\title{
Human Factor in Flight Safety
}

\author{
Hervé Poussin ${ }^{1}$, L. Rochas ${ }^{1}$, T. Vallée ${ }^{1}$, and R. Bertrand ${ }^{2}$ \\ 1. Centre National d'Etudes Spatiales, Guiana Space Center, BP 726-97387 Kourou, French Guiana, France; \\ 2. Centre National d'Etudes Spatiales, Centre Spatial de Toulouse, Edouard Belin, 31401, Toulouse, France
}

\begin{abstract}
A chief goal of the launcher design philosophy is to build launchers offering operational efficiency and not that can be flown safely. Moreover, launch operator focuses the mission design on mission success criteria for the payload and often mitigates launch risks. These sole conditions clearly appear to be inadequate to ensure safety during a flight neither to be up to the safety challenges. Flight safety at CNES/CSG (Centre National d'Etudes Spatiales/Centre Spatial Guyanais) is considered to be a full part job, to be performed separately from the launcher mission. Dedicated ground operators, namely safety officers, who are independent from launcher teams, are ultimately responsible for ensuring the safety. During the flight of a launcher, they are in charge of interrupting actively, making use of a flight termination telecommand from ground, the erroneous flight of a launcher before it endangers people or properties. Human factor is therefore of fundamental importance in flight safety at CNES/CSG. After a quick overview of CNES/CSG, this paper, based on the flight safety way of operation and on the safety officers recruitment, instruction, training and certification, aims at declining how the human factor is handled throughout all flight safety activities.
\end{abstract}

Key words: Flight safety, launcher mission, human factors.

\section{A Quick Overview of the Guyana Space Centre (CNES/CSG)}

CNES/CSG (Centre National d'Etudes Spatiales/Centre Spatial Guyanais) is located near the equator ( $5^{\circ}$ north latitude) in French Guyana, north of Brazil, South America (Fig. 1).

It offers a wide $\left(\sim 100^{\circ}\right)$ opening over the Atlantic Ocean and is well adapted to a great variety of missions from northern to eastern trajectories.

Arianespace, as launch operator, operates three launchers from CNES/CSG: Ariane 5 (the historical European launcher) and, since 2011, Soyuz (Russia) and Vega (Italy) performing 10 to 12 launches per year (Fig. 2).

CNES/CSG, in support to Arianespace, operates the launch range facilities and, by appointment of the French government ensures flight safety for the three rockets Ariane 5, Soyouz and Véga in compliance with the French law for space operations.

Corresonding author: Hervé Poussin, Ph.D.; research fields: flight launcher, and flight safety .

\section{Flight Safety Way of Operation}

The flight safety mission starts at lift off and ends at confirmation that the upper stage is satellised in a stable orbit or at the end of upper stage reentry, when applicable.

The flight safety operations are split into two phases run sequentially along time during a flight:

The 1st phase starts at lift off and ends at the expected LOS (loss of signal) of telecommand stations (all stations being located in French Guyana) or as soon as the launcher ballistic impact point encounters lands.

This phase, called MSI (Mission Sauvegarde et d'Intervention), lasts 5 to $8 \mathrm{~min}$, depending on the launcher considered (Fig. 3).

This 1st phase is both safety critical, as the launcher has a maximum of energy and public areas (at beginning of the flight) are close to the nominal trajectory, and safety active as the flight safety officer can interrupt an erroneous flight by sending a telecommand from ground.

The 2nd phase, called MSA (Mission Sauvegarde et 
Fig. 1 CNES/CSG location.
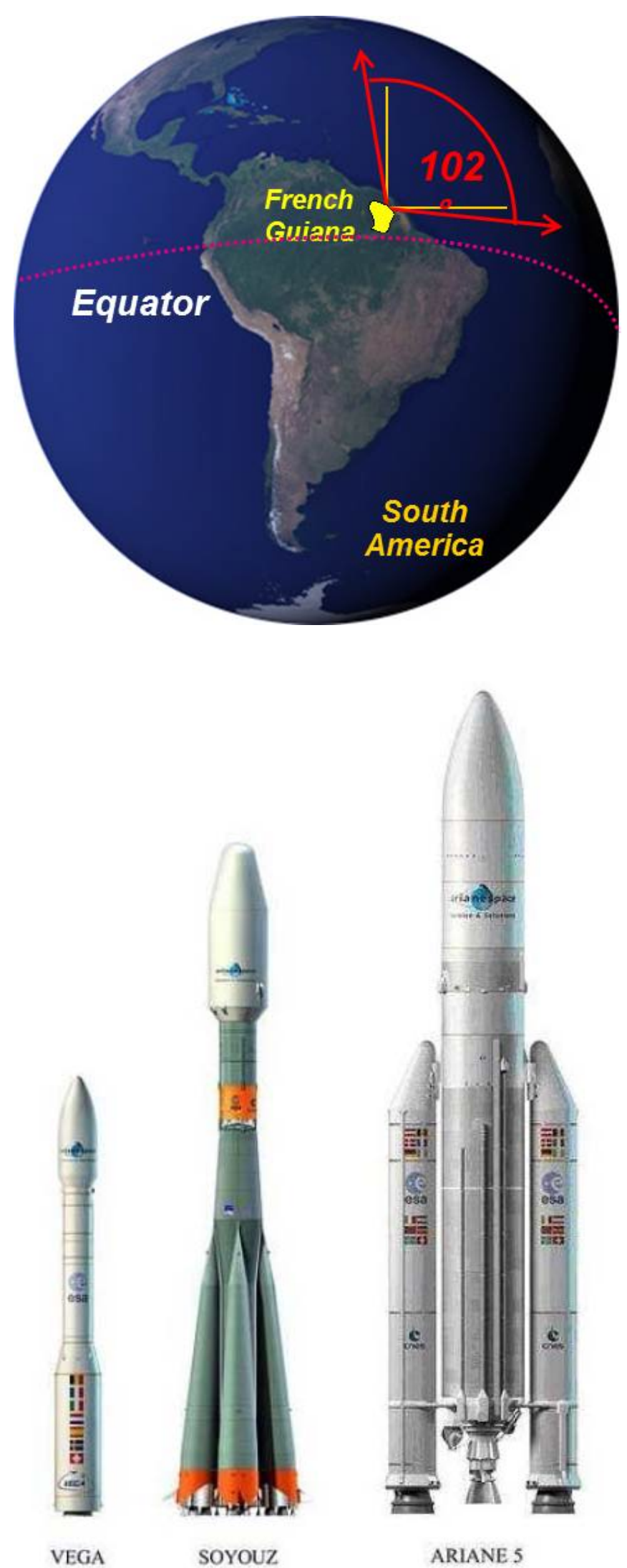

Fig. 2 Launchers operated from CNES/CSG. 
d'Alerte), comes immediately after the MSI and lasts until the end of the safety mission (Fig. 4).

This 2nd phase is safety passive as flight safety performs a monitoring of the flight and a near real time assessment of the fall down areas of the various stages. It can last hours, depending on the mission.

During this phase, flight termination from ground telecommand is forbidden (and not possible) and the associated launch risk is managed, before the launch, through a statistical approach based on launcher failure probability and population density on ground.

Obviously, the 1st phase (the MSI, safety active) of the safety mission is the most important in terms of human factor [1].

Before the flight, and based on the planned nominal trajectory, flight safety defines a safety qualified flight corridor for the launcher. This safety corridor (Fig. 5) is compatible with launcher mission and ensures that people and properties are safe (protected against thermal flow, overpressure, projection of debris and toxicity) whatever the launcher failure scenario occurs.
During the flight, should the launcher cross the limit of this safety corridor, the safety officer will send a flight termination command from ground.

The three-dimension trajectory is displayed on two monitors; one for the impact point (horizontal plane) and the other one for the altitude against the distance to the launch pad (vertical plane). These displays show the nominal trajectory and the alert and safety limits, allowing detecting rapidly a launch vehicle that gets out of respectively its flight corridor and may become dangerous. Two safety officers have the same type of displays but they use two different localization facilities to protect against single point failure.

The flight termination decision follows precise operational criteria that must be identified and applied in real time during the flight, taking into account not only the contingency itself but also any degraded performance or failure of the ground safety facilities, including humans. These criteria are based primarily on the trajectory of the vehicle. They differ for Ariane 5, Soyuz and Vega, due to the specific characteristics and neutralization mode of these launchers.

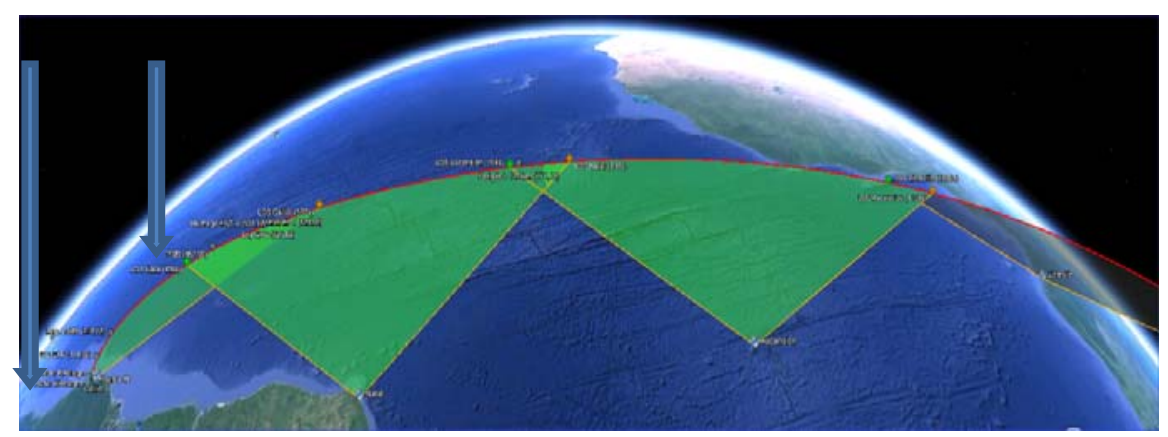

Fig. 3 MSI illustration.

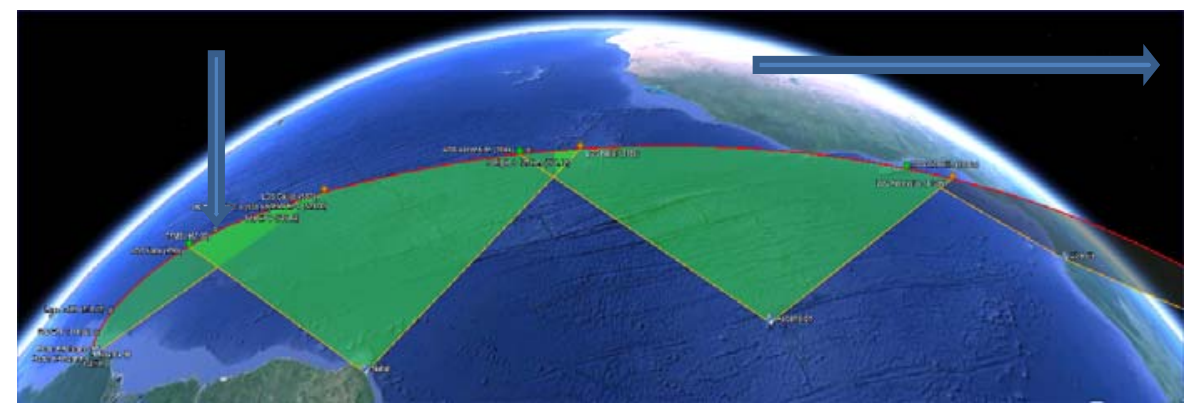

Fig. 4 MSA illustration. 


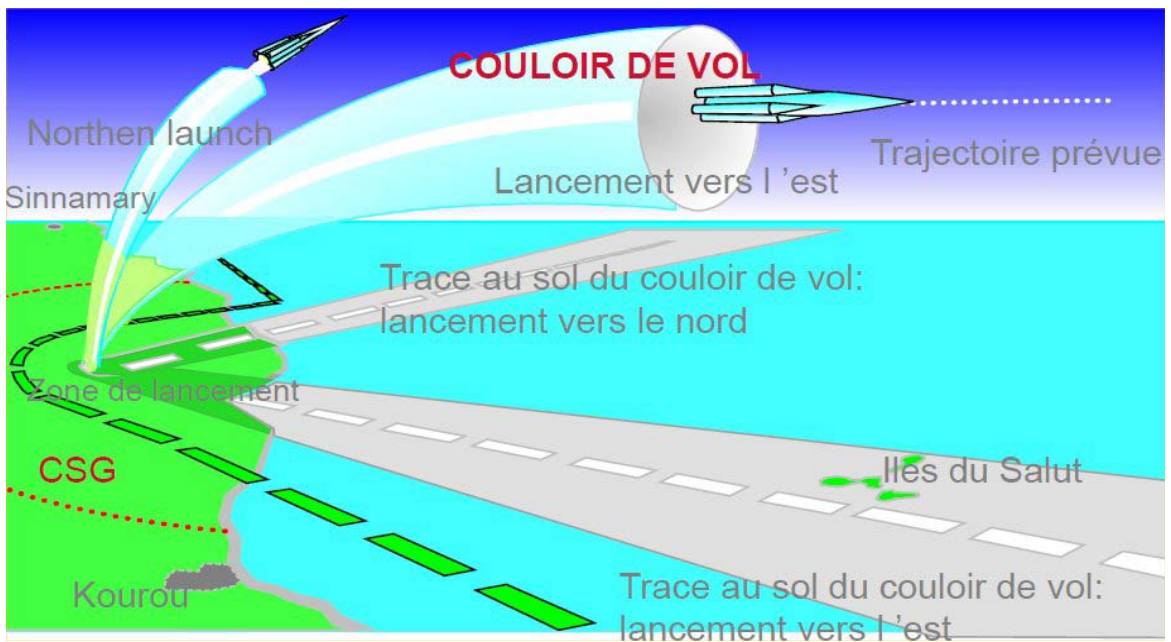

Fig. 5 Safety corridor illustration.

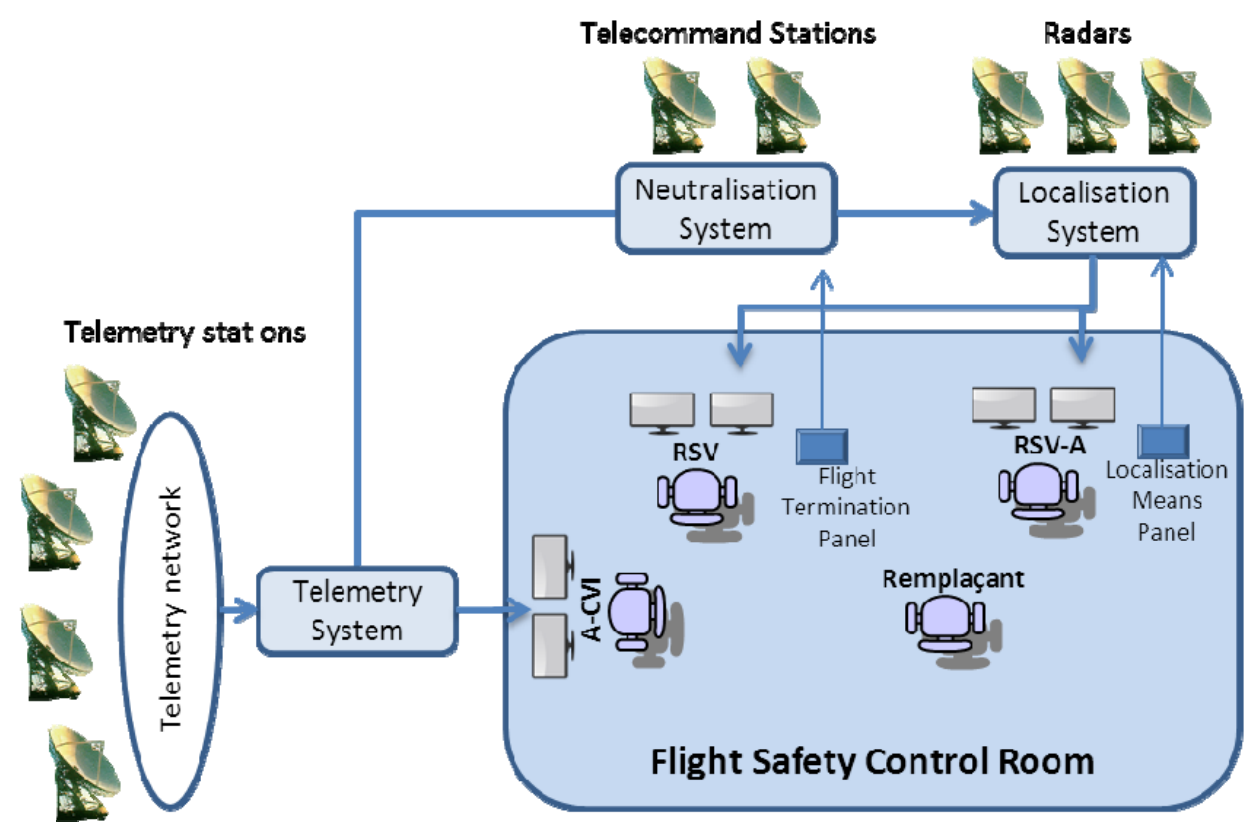

Fig. 6 Flight safety room.

Safety decisions and actions are made in real time by a team of four safety officers, located in a dedicated safety room (Fig. 6), fully independent from the Jupiter mission control centre.

They exchange real time status at voice as follows:

ACVI (telemetry officer) monitors the telemetry received on ground to make early warnings of any launcher failure (propulsion, occurrence of separation events, on board computer and navigation status and status of the on board flight safety algorithm when applicable) and to monitor telecommand link quality RSVA (deputy safety officer) manages the localization facilities and monitors the trajectory of the launcher. He warns about any deviation of the launcher from the nominal trajectory and supports the RSV in his decisions.

RSV (chief safety officer) manages the telecommand facilities, confirms the trajectory deviation, compares it to the flight corridor and sends the flight termination telecommand if needed.

Remplaçant is also present as back up operator and can substitute in real time any of the 3 other operators.

In addition for Vega, an additional (5th) operator monitors the first 25s of the flight with a specific 
algorithm to detect quick deviations of the launcher.

From this way of operation, securing as far as possible the safety team reactions and time to react, launcher and ground facilities failure diagnostics and decision making appear to be of critical importance to avoid wrong, early or late decision.

This is achieved through: clear, simple and robust flight management procedures and flight termination criteria.

Safety criteria are fully based on the launcher localization. This represents one macroscopic, physical and simple parameter, easy to manage in real time and not confusing as can be the numerous on board and on ground device failures and associated failure mode analysis.

FO (fail operational) criteria applied to both safety facilities (on board and on ground) and operators.

This requires to have full redundancy with no single point failure in safety facilities, a remplaçant operator that can substitute any member of the team, and confirmation of safety diagnostic between chief officer and deputy officer as a consequence of the mandatory "confirm or stop an iterate” principle.

The application of the nominality principle that protects launcher from safety device failures. This principle states that if the localization of the launcher is nominal with one localization facility, a deviation observed simultaneously with another localization facility is false and the associated facility is to be considered out of order.

Full independency between on board safety devices and launcher navigation facilities, in particular, launcher localization must be performed using 2 different sensors: one of them being a radar on ground.

Standard communication protocol and short, and precise dialogues are shared among all the safety team members.

Clear, simple and understandable MMIs (man machine interface) are to display decision making information only using a very limited set of colors (e.g., alarms in red, nominal in green, etc.).

Based on the experience gained over last decades and few hundred flights, this way of operation with human deeply involved in the safety loop is proved to be both well under control and efficient with human's strength over the machine lying in its ability to adapt to an unknown situation.

\section{Safety Officers Selection, Instruction, Training and Certification}

In the flight safety way of operation at CNES/CSG, human operators play a key role and therefore special attention is to be paid to selection, instruction, training and certification of safety officers.

This process is clearly defined and controlled and has been fine-tuned over many years.

It starts with the selection of a candidate: this is based on good technical background in engineering together with "human qualities", such as team spirit, decision-making ability, cold blood, stress resistance, etc.

Then the process "instruction, training and certification" applies with the following characteristics:

- It is mandatory for all;

- It is sequentially ordered by increasing level of operational responsibility: starting from ACVI operational position then continuing with the RSVA, RSV, Remplaçant positions and finally ending with the instructor position;

- It is practical: as soon as 2 months after arrival, the safety officer is regularly put in operational situation during training sessions making use of a flight simulator (EMUL, Fig. 8).

Training session typically consists in a succession of 10 simulated flights (or "simulations") built from a combination of pre-recorded malfunction scenarios for the launcher (trajectory or equipment malfunction), the telemetry, telecommand and the localization ground facilities. Each simulation re-creates as closely as possible the conditions of a launch, from typically 
one minute before lift-off up to the simulated neutralization of the launcher or up to the end of the MSI phase. The sessions are supervised by an instructor, who assesses each participant and provides feedback during a short debrief taking place after each simulated flight.

It is adapted to the three launchers operated from CNES/CSG: Ariane 5 instruction process is the backbone for other launchers instruction (Fig. 7) process and additional launcher dediced modules and training sessions are organized for Soyuz and Vega launchers.

It is monitored: certification to an operational position is acquired after the candidate has:
- passed an oral examination to validate the acquisition and understanding of the "theoretical corpus” (position specific operations, operational organization and interfaces, CSG means, intervention criteria, flight safety tools, etc.);

- passed 3 to 6 trainings (depending on the position). This step is required to assess the aptitude to hold the position and allows to take part to a first launch campaign;

- participated to a launch campaign (final validation in real conditions);

It is coached during all the processes by a dedicated safety officer, a senior instructor and the head of the flight safety department.

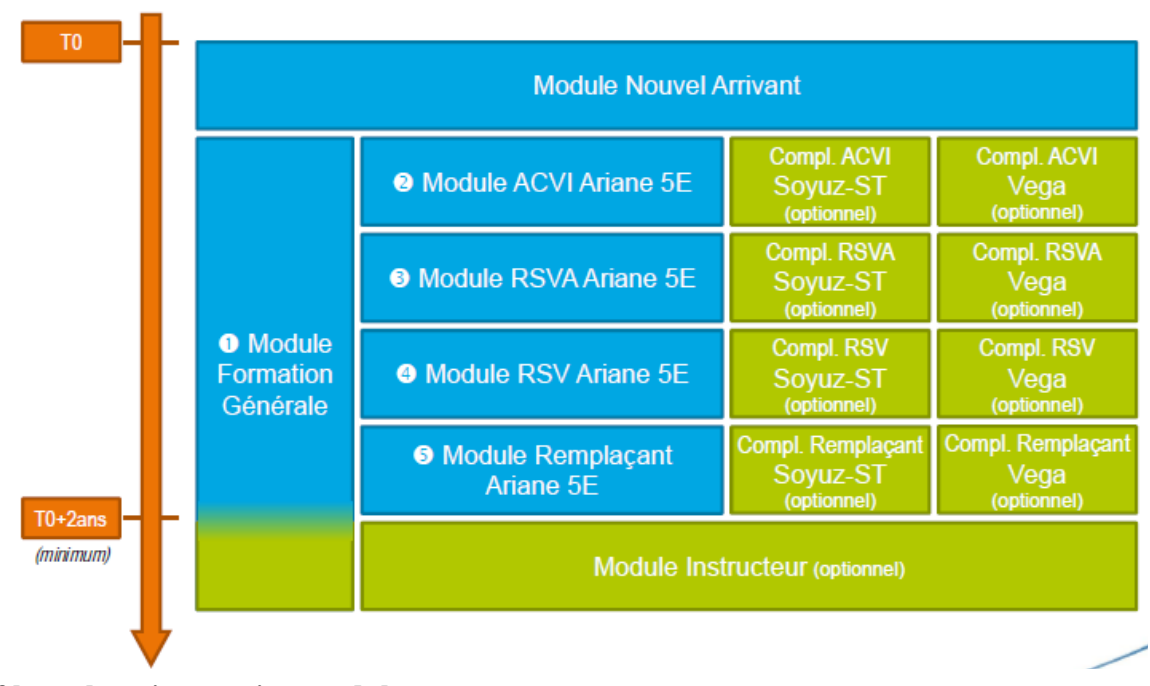

Fig. 7 Mapping of launchers instruction modules.

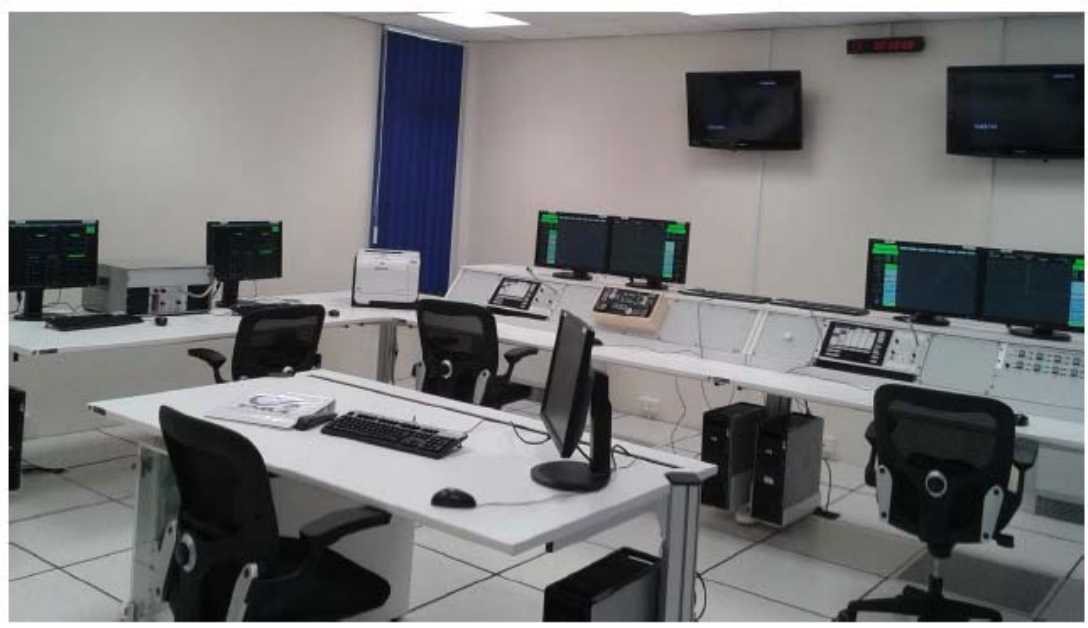

Fig. 8 Flight safety simulator (EMUL). 
It is continued along time: certification for an operational position is valid for 6 months and time validity extension requires participation to refresher training session.

\section{Conclusions}

Based on the experience gained over last decades and few hundred flights, the safety way of operation in place at CNES/CSG, with human deeply involved in the safety loop, has proved to be both well under control and efficient.

It is nevertheless to recognize:

- the non-negligible effort for recruitment, learning and training of safety officers;

- and, with humility, that human errors are inevitable and therefore it is unreasonable to expect error-free human performance.

Otherwise, considering rapid gains in technology and data processing science, a continuous and interesting debate is on going about how to best integrate human with machine, or even how to replace human by machine.

For the time being, the human's strength over the machine lies on human ability to adapt to an unknown situation.

But for how long?

\section{References}

[1] ICAO (International Civil Aviation Organization). 1989. Human Factors Digest No 1. Fundamental Human Factors Concepts. Circular 216-AN/131. ICAO (Montreal, Canada), 1989. 